\title{
CAMBridge
}

\section{Cultural Evolution}

People's Motivations are Changing, and Reshaping the World

RONALD F. INGLEHART, UNIVERSITY OF MichigAN, ANN ARBoR

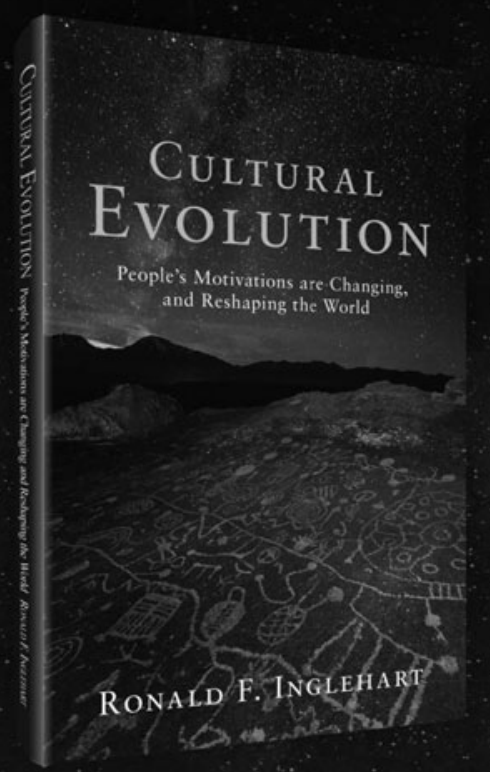

'Cultural Evolution culminates. a remarkably productive halfcentury's exploration of cultural change by Ronald F. Inglehart. This renowned scholar now extends the reach of his theory to global history, while honing his concepts to dissect, for example, the emergence of right-wing: populism and LGBTQ activism. This is Inglehart at his most ambitious and most astute. It is a powerful book.'

Robert D: Putnam Harvard University

For more information, visit Cambridge.org/Inglehart 


\section{Notes to Contributors / Avis aux collaborateurs}

Authors are requested to submit an electronic version of their manuscript, including an abstract of 100 words, in MS Word or RTF, to the JOURNAL's manuscript tracking system at http://www. editorialmanager.com/cjps-rcsp. Manuscripts should be submitted as a single electronic file and be no longer than 8,000 words (all material included) in 12-point type. Book reviewers are asked to ensure reviews do not exceed an 850 word cap. We also encourage an engagement with the key theoretical, methodological and ideological approaches used by book author(s) where this is appropriate rather than a purely descriptive overview. The Journal follows an "in-text" citation style. All material including endnotes and references must be double-spaced. In preparing a manuscript for consideration by the Editorial Board, authors should follow the JOURNAL's style sheet which can be found at the same webpage. Otherwise, the most recent issue of the Journal can serve as a style guide. Manuscripts will not be returned.

Les auteurs-trices doivent soumettre leur manuscrit de format MS Word, qui comprend un résumé de 100 mots, par le biais de la plateforme de suivi de la REVUE Editorial Manager disponible depuis la page Web suivante : http://www. editorialmanager.com/cjps-rcsp. Les manuscrits ne doivent pas dépasser 8000 mots, avec une police de caractère de 12 points. Les auteurs-trices de recension sont invité-es à soumettre un document écrit qui ne dépasse pas 850 mots. Nous encourageons également les auteurs-trices des recensions à lier leur analyse aux principales approches théoriques, méthodologiques et idéologiques utilisées dans le texte, lorsqu'approprié. Les tableaux et les graphiques doivent être soumis sur des pages séparées. Avant de soumettre un manuscrit, les auteurs-trices doivent s'assurer que le protocole de rédaction de la REVUE est respecté. Ce protocole est disponible sur le site Web de la REVUE.

The Editorial Board considers manuscripts on an exclusive basis only; that is, a manuscript submitted for consideration must not have been previously published, nor be under consideration, nor accepted for publication, elsewhere. The Board may judge manuscripts unsuitable if they are too narrow or specialized for an omnibus political science journal.

Le comité de rédaction considère que les manuscrits lui sont soumis en exclusivité; c'està-dire qu'un manuscrit soumis ne doit pas avoir déjà été publié, soumis ou accepté pour publication dans une autre revue. Le comité peut considérer inadmissibles des manuscrits portant sur des sujets trop spécialisés ou trop étroits.

Correspondence in English concerning manuscripts should be addressed to Brenda O'Neill, University of Calgary, Alberta: Email: bloneill@ucalgary.ca

On doit adresser toute communication en français au professeur Mélanie Bourque, Université du Québec en Outaouais, Gatineau, Québec; Courriel: melanie.bourque@uqo.ca

INDEXED IN / INDEXÉE AU : International Political Science Abstracts/Documentation politique internationale; CSA Political Science \& Government: A Guide to the Periodical Literature; ABCCLIO: Historical Abstracts; ABC-CLIO America: History and Life; Political Science Abstracts; Point de Repère : index analytique d'articles de périodiques de langue française; Academic Abstracts; Social Science Source; Social Sciences Index; Sociological Abstracts (partial/ partiel); EBSCO: Academic Search Premier; United States Political Science Documents; Arts \& Humanities Citation Index (A\&HCI); Current Contents/Social and Behavioral Sciences; Social Sciences Citation Index; International Bibliography of the Social Sciences; Social Scisearch; Research Alert; PAIS Bulletin and PAIS Foreign Language Index; Canadian Periodical Index/ Index de périodiques canadiens; Canadian Magazine Index and available on-line in the Canadian Business and Current Affairs Database; Fachinformationsverbund, Internationale Beziehungen und Länderkunde, Freie Universität Berlin; Electoral Studies; Index to Canadian Legal Literature/Index à la documentation juridique au Canada (partial/partiel); Index to Canadian Legal Periodical Literature (partial/partiel); Indian Book Review Digest (partial/ partiel); International Political Science Information Service; IBZ (Internationale Bibliographie der Zeitschriftenliteratur); IBR (Internationale Bibliographie der Rezensionen). 


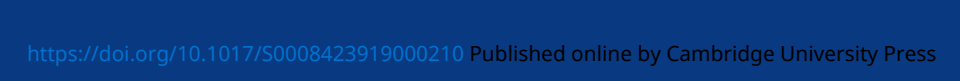

